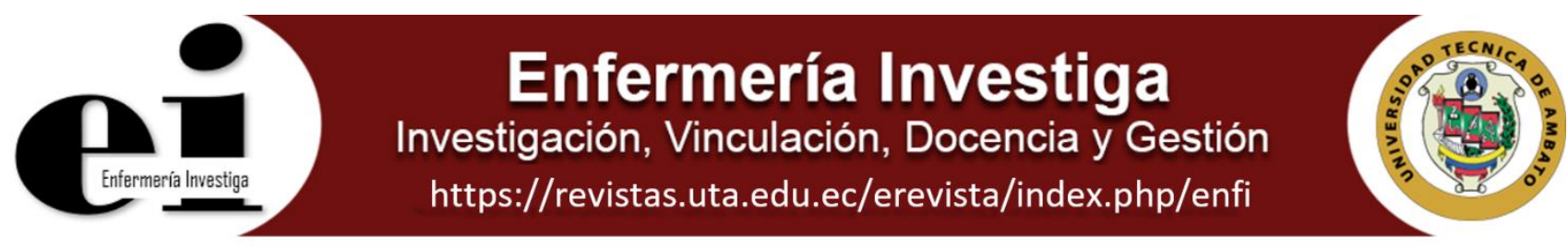

\title{
HIV Y TOXOPLASMOSIS CEREBRAL A PROPÓSITO DE UN CASO
}

\section{HIV AND CEREBRAL TOXOPLASMOSIS ON A CASE}

\begin{abstract}
Jeaneth Naranjo ${ }^{1,2}$ http://orcid.org/0000-0003-4779-5886, Andrés Mora ${ }^{2}$ http://orcid.org/0000-0001-6947-0842 , Patricia Chacha ${ }^{2}$ http://orcid.org/0000-0003-2780-3471, José Luis Herrera ${ }^{3}$ http://orcid.org/0000-0002-6061-0156, Beatriz Venegas ${ }^{3,4}$ http://orcid.org/0000-0002-12707113
\end{abstract}

${ }^{1}$ Docente de la Carrera de Medicina de la Universidad Técnica de Ambato, Ecuador ${ }^{2}$ Hospital General Docente de Ambato, Ecuador

${ }^{3}$ Docente de la Carrera de Enfermería de la Universidad Técnica de Ambato, Ecuador

${ }^{4}$ Unidad de Atención Integral de HIV del Hospital General Docente Ambato, Ecuador

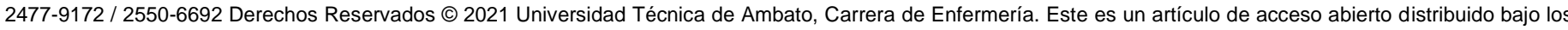

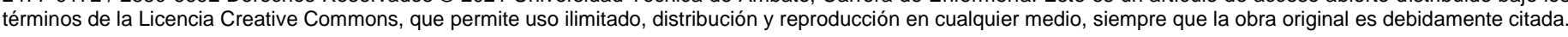

\section{RESUMEN:}

Introducción: En Ecuador al igual que en el resto del mundo la infección por virus de inmunodeficiencia humana $(\mathrm{VIH})$ constituye un problema de salud pública, con diagnósticos tardíos en fase avanzada del síndrome de inmunodeficiencia adquirida (SIDA), con toxoplasmosis cerebral una de las infecciones oportunistas más frecuentes que ocurre en pacientes con linfocitos $C D 4<200 / \mu \mathrm{L}$. Objetivo: realizar diagnóstico precoz de toxoplasmosis cerebral en pacientes con SIDA y considerar como una verdadera emergencia a fin de disminuir la mortalidad de los pacientes inmunosuprimidos. Caso clínico: paciente de género masculino de 31 años, con antecedente de infección por HIV diagnosticado hace 19 días, con convulsiones tónico-clónicas generalizadas de tres meses de evolución, cefalea holocraneana de moderada intensidad, parestesias en hemicara derecha, asimetría comisura labial, tres días antes de su ingreso las crisis convulsivas se hacen diarias, repetitivas y se acompañan de fiebre, al examen físico disartria leve y monoparesia del miembro superior derecho, con biometría hemática con leucocitos $5270 \mathrm{~mm}^{3}$, IgG positiva antiToxoplasma gondii, linfocitosis discreta, pruebas de cuarta y tercera generación para HIV positivas, CD4 de $74 / \mathrm{mm}^{3}$, carga viral de 61500 copias $/ \mathrm{ml}$, resonancia magnética nuclear cerebral contrastada con lesiones intra-axilares corticales, con área hipointensa central, a nivel del lóbulo parietal izquierdo y occipital bilateral, se establece el diagnóstico de encefalitis toxoplásmica, indicándose tratamiento con trimetropin/cotrimoxazol, dexametosona y fármacos antirretrovirales con abacavir-lamivudina y lopinavir-ritonavir teniendo una buena evolución clínica. Conclusiones: El diagnóstico precoz de encefalitis toxoplásmica basado en criterios epidemiológicos, clínicos, de laboratorio y a la resonancia magnética, permitió buena respuesta al tratamiento empírico antitoxoplásmico. Palabras claves: Toxoplasmosis, infecciones oportunistas,
encefalitis

\section{ABSTRACT}

Introduction: In Ecuador, as in the rest of the world, human immunodeficiency virus (HIV) infection constitutes a public health problem, with late diagnoses in an advanced phase of acquired immunodeficiency syndrome (AIDS), with cerebral toxoplasmosis one of the more frequent opportunistic infections that occur in patients with CD4 lymphocytes $<100 / \mu \mathrm{L}$. Objective: to make an early diagnosis of cerebral toxoplasmosis in patients with AIDS and to consider it as a true emergency in order to reduce the mortality of immunosuppressed patients. Clinical case: a 31-year-old male patient, with generalized tonicclonic seizures of three months of evolution, moderate intensity holocranial headache, paresthesia in the right side of the face, asymmetry of the labial commissure, three days before admission the seizures become daily, frequent and accompanied by fever, on physical examination mild dysarthria and monoparesis of the right upper limb, with hematic biometry with leukocytes $5270 \mathrm{~mm} 3$, discrete lymphocytosis, fourth and third generation tests for HIV positive, CD4 of $74 / \mathrm{mm} 3$, viral load of 61,500 copies / ml, brain nuclear magnetic resonance with cortical intra-axillary lesions, with a central hypointense area, at the level of the left parietal lobe and bilateral occipital, the diagnosis of toxoplasmic encephalitis is established, indicating treatment with trimethropin / cotrimoxazole, dexamethasone and antiretroviral drugs with abacavir-lamivudine and lopinavirritonavir having a good clinical evolution. Conclusions: The early diagnosis of toxoplasmic encephalitis based on epidemiological, clinical, laboratory and magnetic resonance criteria, allowed a good response to empirical antitoxoplasmic treatment.

Keywords: Toxoplasmosis, opportunistic infections, encephalitis 


\section{INTRODUCCIÓN}

Los casos de pacientes infectados con VIH se incrementan progresivamente, así en el año 2019 se reportó 38 millones de personas infectadas a nivel mundial (1), en Ecuador Los nuevos casos de VIH/sida, notificados en el año 2020 son 3.823, considerándose un problema de salud pública en Ecuador y el resto del mundo; sin embargo, con la evolución en el diagnóstico y el avance terapéutico con antiretrovirales (ARV) han permitido que los pacientes tengan una mejor calidad de vida, pero tiene gran influencia el diagnóstico precoz, ya que existen casos de pacientes en fases avanzadas del SIDA, con un sistema inmunológico muy comprometido, que debutan con infecciones oportunistas, siendo frecuentes las infecciones a nivel del sistema nervioso como la toxoplasmosis cerebral, que se presenta entre $10-60 \%$ de incidencia $(2,6)$ lo que contribuye a un incremento de la morbimortalidad de estos pacientes $(3,4)$, el diagnóstico temprano y tratamiento específico es fundamental para lograr una óptima recuperación de los pacientes, por estas razones el objetivo de este caso clínico es realizar diagnóstico precoz de toxoplasmosis cerebral en pacientes con SIDA y considerar como una verdadera emergencia a fin de disminuir la mortalidad de los pacientes inmunosuprimidos.

\section{CASO CLÍNICO}

Paciente de 31 años de edad con antecedente de infección por HIV diagnosticado hace 19 días, con cuadro clínico de convulsiones tónico-clónicas generalizadas de tres meses de evolución esporádicas, que se hacen frecuentes en las dos últimas semanas, además parestesias en hemicara derecha, asimetría comisura labial, y cefalea holocraneana de moderada intensidad y tres días antes de su ingreso las crisis convulsivas se hacen diarias, repetitivas y se acompañan de fiebre. Al examen físico signos vitales normales, disartria leve y monoparesia del miembro superior derecho, se decide su ingreso para manejo por especialidad de medicina interna en conjunto con neurocirugía.

En los resultados de las pruebas de laboratorio: biometría hemática con leucocitos $5270 \mathrm{~mm}^{3}$, linfocitosis discreta, hemoglobina y plaquetas normales, procalcitonina negativa. En la Resonancia Magnética Nuclear (RMN) Cerebral contrastada se observa lesiones intra-axilares corticales, con refuerzo en anillo periférico al Galodineo con área hipointensa central, a nivel del lóbulo parietal izquierdo y occipital bilateral (figura 1).

Por las manifestaciones clínicas y el estudio de neuroimagen, se sospecha de una infección oportunista de sistema nervioso central, se decide su ingreso con tratamiento antibiótico empírico en base a ceftriaxona 2 gr vía intravenoso (IV) cada 12 horas, metronidazol $500 \mathrm{mg}$ IV cada 6 horas, vancomicina $500 \mathrm{mg}$ IV cada 6 horas, recibe por 11 días, trimetropin /sulfametoxazol cada 12 horas y dexametosona $8 \mathrm{mg}$ IV cada 12 horas.

Durante la evolución intrahospitalaria se realizan las siguientes pruebas de laboratorio: IgG positiva anti-Toxoplasma gondii, pruebas para el diagnóstico microbiológico de la infección por el VIH: serología positiva de ELISA (Enzyme-Linked ImmunoSorbent Assay) de cuarta y tercera generación para HIV, carga viral del VIH alta de 61500 copias $/ \mathrm{ml}$, CD4 bajos de $74 / \mathrm{mm}^{3}$, con resultados negativos para serología Hepatitis A, B y C negativa, aglutinaciones febriles, baciloscopia de esputo para tuberculosis. En la Espectroscopía por Resonancia Magnética de protón muestra presencia de incremento de lactato y lípidos en moderada cantidad, en relación con probable proceso inflamatorio (figura 2), debido a la evolución clínica y los resultados de pruebas de laboratorio se considera el diagnóstico encefalitis por toxoplasma, se descartó compromiso ocular por toxoplasmosis con un fondo de ojo normal, se suspende antibioticoterapia y se inicia tratamiento con trimetropin/sulfametaxazol 800/160 mg tabletas cada 8 horas desde el 09/01/2020, con una evolución es favorable y el 15/01/2020 se inicia fármacos antirretrovirales con abacavir-lamivudina y lopinavir-ritonavir seis días más tarde es dado de alta en buenas condiciones con continuidad del tratamiento antibiótico y TAR y para su seguimiento y control por especialidad.

Tabla 1. Biometría Hemática

\begin{tabular}{|l|l|}
\hline 31/Dic/19 HEMOGRAMA \\
\hline Leucocitos & 5270 \\
\hline Hematíes & $5^{\prime} 170.000$ \\
\hline Hemoglobina & $14,30 \mathrm{gr} / \mathrm{dl}$ \\
\hline Hematocrito & $46,20 \%$ \\
\hline VCM & 89,3 \\
\hline HCM & 27,7 \\
\hline Plaquetas & 267.000 \\
\hline Neutrófilos\% & $60,10 \%$ \\
\hline Linfocitos \% & $33,50 \%$ \\
\hline Monocitos\% & $4 \%$ \\
\hline Eosinófilos\% & $1,80 \%$ \\
\hline Basófilos\% & $0,60 \%$ \\
\hline
\end{tabular}


Tabla 2. Carga Viral/CD4

\begin{tabular}{|l|l|l|}
\hline Fecha & CARGA VIRAL & CD4 \\
\hline $10 / 1 / 2020$ & 61500 & \\
\hline $11 / 1 / 2020$ & & 74 \\
\hline
\end{tabular}

Fuente: Archivo del Historia clínica Hospital General Docente Ambato (HGDA)

Figura 1 RMN cerebral contrastada
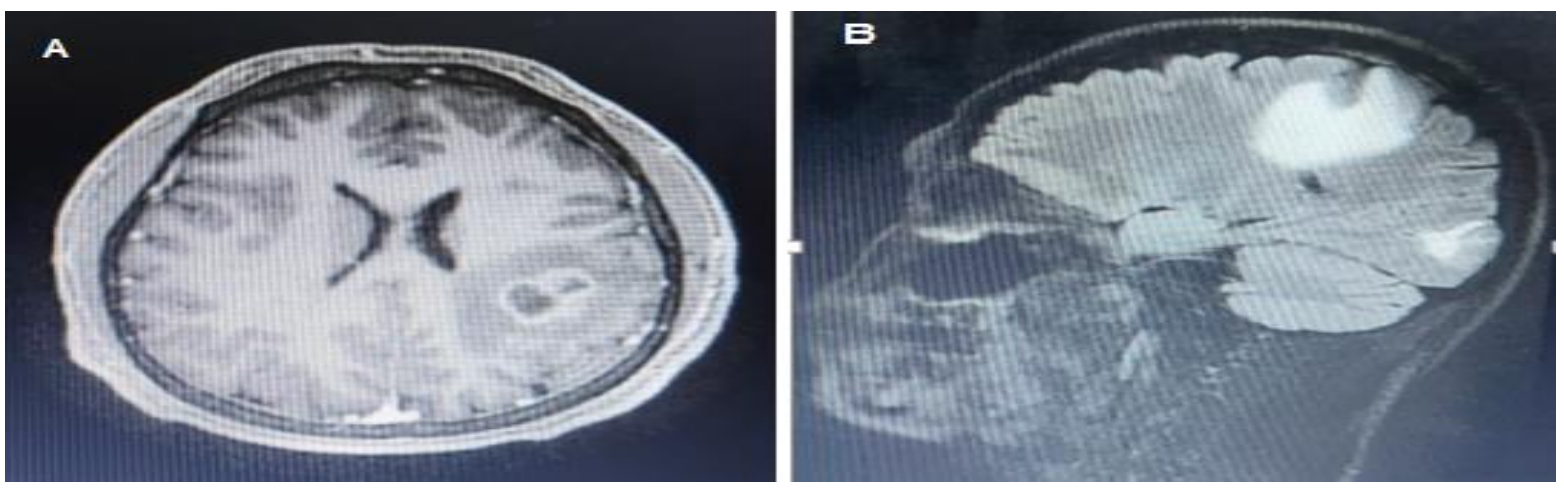

Fuente: Archivo del Historia clínica Hospital General Docente Ambato (HGDA)

RMN Cerebral: A) Corte axial $\quad$ B) Corte sagital

RMN T1 con contraste se observa lesiones intra-axilares corticales, con refuerzo en anillo periférico al Galodineo con área hipointensa central, a nivel del lóbulo parietal izquierdo y occipital bilateral.
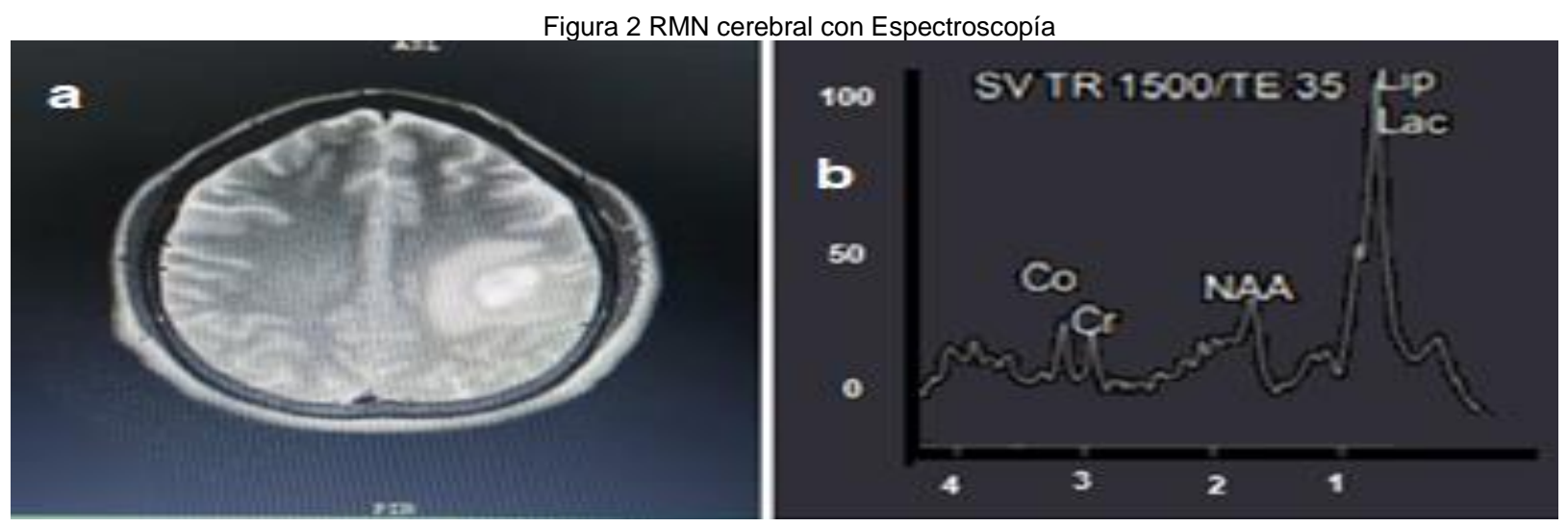

Fuente: Archivo Historia clínica Hospital General Docente Ambato: a) Presencia de lesiones intra-axilares corticales nivel de lóbulo parietal izquierdo, edema sustancia blanca peri lesiones de predominio izquierdo. b) Espectroscopia muestra presencia de incremento de lactato y lípidos en moderada cantidad, en relación con probable proceso inflamatorio (Co: colina; Cr: creatina, NAA: N acetil aspartato; Lip: lípidos; Lac lactato.)

\section{DISCUSIÓN}

La toxoplasmosis es una zoonosis causada por el parásito intracelular el Toxoplasma gondii que se encuentra hospedado en varios animales y es a través la ingestión de ooquistes de las heces que contaminan al ser humano y la ingestión de carne mal cocida que contenga quistes, En el ser humano, además de la transmisión por ingestión de los ooquistes del suelo procedentes de los gatos, existe una segunda vía de transmisión que se hace a través de los tejidos con quistes de los animales que le sirven de alimento, como cerdo, bovinos, ovejas, aves, etc $(4,5$,$) . con mayor susceptibilidad en los pacientes inmunocomprometidos, particularmente$ aquellos con VIH/SIDA, que presentan frecuentemente toxoplasmosis cerebral, con una incidencia del 10 al $50 \%,(1,2,8,9)$ dato que 
no se relaciona la casuística en la Unidad de Atención Integral Hospital General Docente Ambato (HGDA), Ecuador hasta el mes de Julio de un total de 421 pacientes el $0,7 \%$ (3 pacientes) tuvieron encefalitis toxoplásmica, de estos pacientes, dos fallecieron por las condiciones generales en las que ingresaron.

La clínica de cefaleas, parestesias faciales, crisis convulsivas (6-8) son frecuentes en afectaciones del sistema nervioso central de tipo infecciosos como los abscesos, encefalitis y/o meningitis, o procesos neoplásicos como linfomas. Como parte del diagnóstico pero en el contexto de un paciente con HIV, con la clínica del paciente como en el caso presentado en donde la manifestación principal fue las convulsiones tónico-clónicas y hemiparesias faciales derechas y con el diagnóstico previo de HIV, se plantea el diagnóstico de la posible infección del sistema neurológico, considerando que el parásito tiene un trofismo mayor hacia las células de sistema nervioso central como astrocitos y microglía que pasan a ser células diana para el toxoplasma y que interfiere en la inmunidad y su respuesta ante infecciones $(8,18,20)$. Se debe considerar también las lesiones en neuroimagen, en la RMN inicial con la que ingresó el paciente se observa imágenes sugestivas de absceso versus toxoplasmosis, siendo necesario confirmar el diagnóstico por lo que se hizo exámenes de laboratorio.

En cuanto al diagnóstico se confirma en el paciente la infección por HIV con pruebas de tercera y cuarta generación y la medición de carga viral (61.5009 y de CD4 (74) determinan que es un paciente con SIDA (CD4 < a 200)(8,10), en cuanto a las pruebas de anticuerpos para toxoplasma se conoce que la lgM asciende la primera semana y desciende en uno a dos meses y la lgG, se ve en pacientes expuestos al parásito, también se eleva en fase aguda, $(9,10)$ por lo tanto no es certero el diagnóstico. Se considera lgG en fase aguda cuando en un lapso de tres semanas se hace un comparativo elevándose esta, actualmente se tiene más pruebas moleculares que podrían contribuir al diagnóstico de toxoplasmosis, sin embargo, el no tener al alcance estas, así como la falta de estandarización de las mismas hace que los resultados varíen entre sí. $(9,19,21)$, es importante mencionar que para confirmar el diagnóstico se debe realizar pruebas histopatológicas o investigar material nucleico en líquido cefalorraquídeo, situación que no se pudo realizar en el paciente actual $(8,22,23)$. De allí la necesidad de emplear otros métodos; $y$ actualmente la neuroimagen contribuye a ello $(11,12,22)$. La Tomografía cerebral y la RMN contrastadas son ideales para determinar lesiones, generalmente se observa la imagen doble anillo: hipodensidad central con bordes con realce circular o lesiones hiperdensas con realce asociado a edema vasogénico producto de la fagocitosis en los procesos inflamatorios. $(12,13,14)$. Pero es la RMN con espectroscopia es una técnica que analiza el comportamiento metabólico de los tejidos sanos o con alguna patología, indica con precisión las lesiones y su localización $(11,14)$.

La encefalitis por toxoplasmosis constituye una verdadera emergencia por lo que acertadamente desde su ingreso se inicia tratamiento con antibioticoterapia empírica: ceftriaxona, vancomicina, metronidazol, trimetoprim-sulfametoxazol (TMP-SMX), esquema adecuado para cubrir un absceso o un proceso infeccioso de sistema nervioso. Con el diagnóstico establecido se da (TMPSMX 800/160 mg cada 8 horas a más del tratamiento TAR, el esquema de elección en toxoplasmosis cerebral es la pirimetamina más sulfadiazina (15-17), un segundo esquema muy eficiente es TMP-SMX, es el establecido en el caso presentado, que al ser un paciente inmunosuprimido debe continuar con el mismo antibiótico como profilaxis a fin de evitar otras infecciones hasta que su CD4 aumente como respuesta al tratamiento TAR $(24,25)$.

Desde un punto de vista investigativo docente consideramos que este caso contribuye a que los profesionales concienticen sobre la realidad de las infecciones del sistema nervioso central en pacientes inmunodeprimidos porque puedes ser potencialmente mortales, siendo importante llegar a un diagnóstico oportuno para que el paciente no se deteriore y se recupere sin complicaciones, pero fundamentalmente es la prevención.

\section{REFERENCIAS}

1. Organización Mundial de la Salud.- VIH/SIDA.- Sitio web mundial. 6 Julio2020.-

Disponible en https://www.who.int/es/news-room/fact-sheets/detail/hiv-aids

2. Farfán Cano GG, Troya Toro M, Herrera Álava J. Toxoplasmosis cerebral en paciente VIH. Revista científica digital INSPILIP. Ecuador 2018;2(2):1-8, DOI:10.31790/inspilip.v2i2.52.g56, Disponible en: http://www.inspilip.gob.ec/

3. Velastegui M, Touriz MA, Pérez L. Estudio retrospectivo de toxoplasmosis cerebral en pacientes con sida. Revista Científica de Investigación actualización del mundo de las Ciencias. (RECIAMUC). Octubre- 2017;1(4):566-77. DOI: https://doi.org/10.26820/reciamuc/1.4.2017.566-577

4. Lara-Palacios IA, Canché-Chi V, Ortiz-Zolozabal P. Toxoplasmosis cerebral en un paciente con recién diagnóstico de VIH/SIDA. Salud Quintana Roo. 1 de abril de 2020;11(39):16-20. Disponible: https://www.medigraphic.com/cgibin/new/resumen.cgi?IDARTICULO $=92489$

5. Medina J. Pautas para el diagnóstico y tratamiento de las infecciones oportunistas en pacientes con VIH. Univ Republica Urug. julio de 2016;203. Disponible: http://www.infectologia.edu.uy/images/archivos/manual-hiv2016.pdf

6. Duran E, Mirazo I, Combol A. Toxoplasmosis cerebral en pacientes con SIDA. Parasitología al día. julio de 1997;21(3-4):123128. http://dx.doi.org/10.4067/S0716-07201997000300011

7. Alonso NEV, Morales OQ, Patricio RG. Neurotoxoplasmosis como debut de paciente con SIDA. Acta Médica Cent. 2010;4(4):41-47. Disponible: http://www.revactamedicacentro.sld.cu/index.php/amc/article/view/534/700

8. Lasso B M. Diagnóstico y tratamiento de infecciones oportunistas en el paciente adulto con infección por VIH/SIDA. Rev Chil Infectol. Octubre de 2011;28(5):440-460. Disponible: http://dx.doi.org/10.4067/S0716-10182011000600010

9. Quevedo MC, Cruz AH, Contreras AJD. El nexo entre biología, respuesta inmune y clínica en la infección por Toxoplasma gondii. Rev Cuba Investig Bioméd. 3 de octubre de 2019;38(4). Disponible en: http://scielo.sld.cu/scielo.php?script=sci arttext\&pid=S0864-03002019000400014\&lng=es\&nrm=iso

10. Martínez E, Arribas JR, Polo R, García JG, Palacios R. Documento de Consenso de SIDA/Plan Nacional sobre el sida respecto al tratamiento antirretroviral en adultos infectados por el virus de la inmunodeficiencia humana. GESIDA. Febrero 
de 2020;145 Disponible en: $\underline{\text { https://gesida-seimc.org/wp- }}$

content/uploads/2020/07/TAR GUIA GESIDA 2020 COMPLETA Julio.pdf.

11. Sganbi N.- Infecciones del sistema nervioso: Nuevas herramientas diagnósticas.- Revista Argentina de Radiología. Febrero 2015. [Internet]. [citado 20 de Junio de 2020]. Disponible en: https://www.elsevier.es/es-revista-revista-argentina-radiologia383-pdf-S0048761915000034

12. Valencia E. Lesiones cerebrales ocupantes de espacio con efecto de masa en los pacientes con infección por VIH: diagnóstico diferencial entre la toxoplasmosis cerebral y el linfoma cerebral primario.- Revista Multidisciplinar del SIDA. Madrid abril 2020;8(20). Disponible en: https://www.revistamultidisciplinardelsida.com/lesiones-cerebrales-ocupantes-deespacio-con-efecto-de-masa-en-los-pacientes-con-infeccion-por-VIH-diagnostico-diferencial-entre-la-toxoplasmosiscerebral-y-el-linfoma-cerebral-primario/

13. Miranda G G, Díaz G C, Dellien Q H, Hermosilla H. Enfrentamiento imagenológico en las lesiones cerebrales en pacientes VIH. Revista Chilena de Radiología. 2008;14(4):200-207. Disponible en: http://dx.doi.org/10.4067/S071793082008000400004

14. Zambrano CA, Rosero LY, Limones MS, Campaña MY, Parra JA. Un acercamiento a la toxoplasmosis cerebral y su diagnóstico imagenológico. RECIMUNDO. 19 de marzo de 2019; 3(1):1558-1578. DOI: https://doi.org/10.26820/recimundo/3.(1).enero.2019.1558-1578

15. Dedicoat M, Livesley N.-Tratamiento de la encefalitis toxoplásmica en individuos con infección por VIH / especialmente en ámbitos de bajos recursos).- (Revisión Cochrane traducida). En: La Biblioteca Cochrane Plus, número 3, 2008. Oxford, Update Software Ltd.DOI: DOI: 10.1002/14651858.CD005420.pub2 Disponible en: http://www.update-software.com.

16. Desse J. SADI - Sociedad Argentina de Infectología - Recomendaciones sobre el Manejo de Infecciones Oportunistas en Pacientes con infección por HIV. [citado 28 de agosto de 2020]; Disponible en: https://www.sadi.org.ar/guiasrecomendaciones-y-consensos/item/392-recomendaciones-sobre-el-manejo-de-infecciones-oportunistas-en-pacientescon-infeccion-por-hiv.

17. Hernandez AV, Thota P, Pellegrino D, Pasupuleti V, Benites-Zapata VA, Deshpande A, et al. A systematic review and metaanalysis of the relative efficacy and safety of treatment regimens for HIV-associated cerebral toxoplasmosis: is trimethoprimsulfamethoxazole a real option? HIV Med. 2017;18(2):115-124.DOI: 10.1111/hiv.12402. Disponible en: https://pubmed.ncbi.nlm.nih.gov/27353303/

18. Jose Ernesto Vidal Md, Phd, HIV-Related Cerebral Toxoplasmosis Revisited: Current Concepts and Controversies of an Old Disease, SAGE Journal, Agosto 2019, DOI: 10.1177/2325958219867315, Disponible en: https://doi.org/10.1177/2325958219867315

19. Marie Gladys Robert, Marie-Pierre Brenier-Pinchart, Cécile Garnaud, Hélène Fricker-Hidalgo \& Hervé Pelloux (2021) Molecular diagnosis of toxoplasmosis: recent advances and a look to the future, Expert Review of Anti-infective Therapy, DOI: $\quad 10.1080 / 14787210.2021 .1941867$, Disponible:

https://www.Xmol.com/paper/1403543786787160064?recommendPaper=1311000964206137344

20. Schlüter D, Barragan A. Advances and Challenges in Understanding Cerebral Toxoplasmosis. Front Immunol. 2019;10:242.

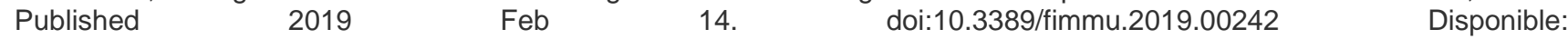
https://www.ncbi.nlm.nih.gov/pmc/articles/PMC6401564/

21. Pathologic and immunohistochemical findings in an outbreak of systemic toxoplasmosis in a mob of red kangaroos Mariano Carossino, Rudy Bauer, Mark A. Mitchell, Charles O. Cummings, Anke C. Stöhr, Nobuko Wakamatsu, Kimberly Harper, Ingeborg M. Langohr, Kendra Schultz, Maria S. Mitchell, Daniel K. Howe, Udeni B. R. Balasuriya J Vet Diagn Invest. 2021 May; 33(3): 554-565. Published online 2021 Mar 19. doi: 10.1177/10406387211001869. Disponible en: https://www.ncbi.nlm.nih.gov/pmc/articles/PMC8120092/

22. Ayele, B. A., Getu, Z., \& Samuel, A. New-onset focal seizure as a presenting feature of HIV infection: a case report and mini review to the challenge in resource-limited settings. AIDS research and therapy. 2021;18(1):12. https://doi.org/10.1186/s12981-021-00344-0

23. Azovtseva, O. V., Viktorova, E. A., Bakulina, E. G., Shelomov, A. S., \& Trofimova, T. N. Cerebral toxoplasmosis in HIVinfected patients over 2015-2018 (a case study of Russia). Epidemiology and infection. 2020;148:e142. https://doi.org/10.1017/S0950268820000928

24. Dunay, I. R., Gajurel, K., Dhakal, R., Liesenfeld, O., \& Montoya, J. G. Treatment of Toxoplasmosis: Historical Perspective, Animal Models, and Current Clinical Practice. Clinical microbiology reviews. 2018;31(4):e00057-17. https://doi.org/10.1128/CMR.00057-17

25. Abbasi Fard, S., Khajeh, A., Khosravi, A., Mirshekar, A., Masoumi, S., Tabasi, F., Hassanzadeh, T., \& Mortazavi, M. M. Fulminant and Diffuse Cerebral Toxoplasmosis as the First Manifestation of HIV Infection: A Case Presentation and Review of the Literature. The American journal of case reports. 2020;21: e919624. https://doi.org/10.12659/AJCR.919624 